\title{
DEFORMING AREA PRESERVING DIFFEOMORPHISM OF SURFACES BY MEAN CURVATURE FLOW
}

\author{
Mu-TaO WANG
}

\begin{abstract}
Let $f: \Sigma_{1} \rightarrow \Sigma_{2}$ be an area preserving diffeomorphism between compact Riemann surfaces of constant curvature. The graph of $f$ can be viewed as a Lagrangian submanifold in $\Sigma_{1} \times \Sigma_{2}$. This article discusses a canonical way to deform $f$ along area preserving diffeomorphisms. This deformation process is realized through the mean curvature flow of the graph of $f$ in $\Sigma_{1} \times \Sigma_{2}$. It is proved that the flow exists for all time and the map converges to a canonical map. In particular, this gives a new proof of the classical topological results that $O(3)$ is a deformation retract of the diffeomorphism group of $S^{2}$ and the mapping class group of a Riemman surface of positive genus is a deformation retract of the diffeomorphism group.
\end{abstract}

\section{Introduction}

The mean curvature flow is an evolution process under which a submanifold evolves in the direction of its mean curvature vector. It represents the most effective way to decrease the volume of a submanifold. The codimension one case of the mean curvature flow has been studied extensively while very little is known in the higher codimension case. The multidimensionality of the normal bundle presents the essential difficulties in such attempts.

[14] studies the mean curvature flow of surfaces in an Einstein four-manifold and proves that a symplectic surface in a Kähler-Einstein surface remains symplectic along the mean curvature flow and the flow does not develope any type I singularity. When the embient four-manifold $M$ is locally a product of Riemann surfaces, there are two Kähler forms $\omega^{\prime}=\omega_{1}-\omega_{2}$ and $\omega^{\prime \prime}=\omega_{1}+\omega_{2}$ that determine opposite orientations for $M$. We proved long time existence and convergence of the mean curvature flow in [14] under the condition that the initial surface is symplectic with respect to both $\omega^{\prime}$ and $\omega^{\prime \prime}$. These results have been generalized to arbitrary dimension and codimension in [15]. This article studies the case when the initial surface is Lagrangian with respect to one Kähler form and symplectic with respect to the other.

Theorem A. Let $M$ be a compact four manifold. If the universal covering of $M$ is any one of $S^{2} \times S^{2}, \mathbb{R}^{2} \times \mathbb{R}^{2}$ or $\mathbb{H}^{2} \times \mathbb{H}^{2}$ and $\Sigma$ is a compact surface in $M$

Received October 19, 2000.

Revised version received April 15, 2001. 
that is Lagrangian with respect to $\omega_{1}-\omega_{2}$ and symplectic with respect to $\omega_{1}+\omega_{2}$, then the mean curvature flow of $\Sigma$ exists smoothly for all time.

It is proved by maximum principle in $\S 2$ that each slice $\Sigma_{t}$ is again Lagrangian with respect to $\omega_{1}-\omega_{2}$ and symplectic with respect to $\omega_{1}+\omega_{2}$. In this Lagrangian case, the condition of being symplectic with respect to $\omega_{1}+\omega_{2}$ is the same as saying $\Sigma$ is locally a graph over both $\Sigma_{1}$ and $\Sigma_{2}$. This happens when $\Sigma$ is the graph of a diffeomorphism between $\Sigma_{1}$ and $\Sigma_{2}$. Recall a diffeomorphism $f: \Sigma_{1} \rightarrow \Sigma_{2}$ is called area preserving if $f^{*} \omega_{2}=\omega_{1}$. It is not hard to see this is true if and only if the graph of $f$ in $M=\Sigma_{1} \times \Sigma_{2}$ is an embedded Lagrangian surface with respect to the symplectic structure $\omega^{\prime}=\omega_{1}-\omega_{2}$.

As for convergence at infinity, we prove the following general subconvergence theorem.

Theorem B. Let $\Sigma_{1}$ and $\Sigma_{2}$ be compact Riemann surfaces with the same constant curvature and $f: \Sigma_{1} \mapsto \Sigma_{2}$ be an area preserving diffeomorphism. As $t \rightarrow \infty$, a sequence of the mean curvature flow of the graph of $f$ converges to a smooth minimal Lagrangian graph.

After this work was completed, the author was informed that K. Smoczyk claimed a proof to this theorem in the non-positive curvature case assuming an extra angle condition.

The limit in this case is a "minimal map". This notion was introduced by R. Schoen in [6].

Definition 1.1. A map $f: \Sigma_{1} \rightarrow \Sigma_{2}$ is called a minimal map if the graph is a minimal embedding in $M$.

Schoen also proved the existence and uniqueness of minimal Lagrangian map when $\Sigma_{1}$ and $\Sigma_{2}$ are hyperbolic surfaces. Theorem B gives a new proof of the existence part.

When $\Sigma_{1}=\Sigma_{2}$ and $f$ is homotopic to identity map, we prove the following uniform convergence theorem.

Theorem C. Let $\Sigma_{1}$ be a compact Riemann surface of constant curvature and $f: \Sigma_{1} \mapsto \Sigma_{1}$ be an area preserving diffeomorphism that is homotopic to the identity map. Denote by $\Sigma$ the graph of $f$ in $\Sigma_{1} \times \Sigma_{1}$. The mean curvature flow of $\Sigma$ converges uniformly to a totally geodesic Lagrangian graph along Lagrangian graphs.

When $\Sigma_{1}$ is a sphere, we prove a stronger convergence in $\S 3$.

The result in [14] has applications in the deformation of maps between Riemann surfaces. It was proved that any map between spheres with Jacobian less than one is deformed to a constant map along the mean curvature flow of its graph. The result in this article applies to the case when Jacobian is equal to one. 
Corollary C. Any area preserving diffeomorphism $f: \Sigma_{1} \mapsto \Sigma_{1}$ that is homotopic to the identity can be deformed to an isometry along area preserving diffeomorphisms by the mean curvature flow.

Since any diffeomorphism is isotopic to an area preserving diffeomorphism, this gives a new proof of Smale's theorem [12] that $O(3)$ is the deformation retract of the diffeomorphism group of $S^{2}$. For a positive genus Riemann surface, this implies the identity component of the diffeomorphism group is contractible.

\section{Long time existence}

Let $M$ be a smooth compact four manifold whose universal covering is any of $S^{2} \times S^{2}, \mathbb{R}^{2} \times \mathbb{R}^{2}$ or $\mathbb{H}^{2} \times \mathbb{H}^{2}$. Equipping with the quotient metric, $M$ is in fact a locally symmetric space. The standard Kähler forms on the factors extend to two parallel forms $\omega_{1}$ and $\omega_{2}$ on $M . \omega^{\prime}=\omega_{1}-\omega_{2}$ and $\omega^{\prime \prime}=\omega_{1}+\omega_{2}$ are two Kähler forms on $M$ that determine opposite orientations for $M$ in the sense that $\omega^{\prime} \wedge \omega^{\prime}=-\omega^{\prime \prime} \wedge \omega^{\prime \prime}$. The metric on $M$ is in particular Kähler-Einstein with respect to either Kähler form and Ric $=c \cdot g$. We shall fix the Kähler structure of $M$ to be $\omega^{\prime}$.

Let $F_{0}: \Sigma \rightarrow M$ be a Lagrangian immersion of a compact surface $\Sigma$. We evolve $F_{0}$ in the direction of its mean curvature vector.

$$
\frac{d F}{d t}(x, t)=H(x, t)
$$

where $F: \Sigma \times[0, T) \rightarrow M$ is a one parameter family of immersions $F_{t}(\cdot)=F(\cdot, t)$ of $\Sigma$ and $H(x, t)$ is the mean curvature vector of $F_{t}(\Sigma)$ at $F_{t}(x)$. Whenever there is no ambiguity, we shall write $F_{t}(\Sigma)=\Sigma_{t}$.

Let $*$ be the Hodge operator on $\Sigma$, then $* \omega_{i}$ is the Jacobian of the projection from $\Sigma$ onto the $i$-th factor of $M$.

Definition 2.1. We say $\Sigma$ is a local graph with respect to $\omega_{i}$ if $* \omega_{i}>0$ on $\Sigma$.

The following proposition shows in particular the condition of being the graph of an area preserving diffeomorphism is preserved and thus the mean curvature flow does provide a deformation for such diffeomorphisms.

Proposition 2.1. Being a Lagrangian local graph in $M$ is preserved along the mean curvature flow.

Proof. The condition is equivalent to $\omega^{\prime}=0$ and $\omega^{\prime \prime}>0$. By Proposition 4.1 in [14] for any parallel Kähler form $\omega, \eta=* \omega$ satisfies the following equation,

$$
\frac{d}{d t} \eta=\Delta \eta+\eta\left[\left(h_{31 k}-h_{42 k}\right)^{2}+\left(h_{32 k}+h_{41 k}\right)^{2}\right]+c \eta\left(1-\eta^{2}\right)
$$

where $\left\{e_{1}, e_{2}, e_{3}, e_{4}\right\}$ is an orthonormal basis for $T_{p} M$ such that $\left\{e_{1}, e_{2}\right\}$ forms an orthonormal basis for $T \Sigma_{t}$. The basis is chosen so that $d \mu\left(e_{1}, e_{2}\right)>0$ and $\omega^{2}\left(e_{1}, e_{2}, e_{3}, e_{4}\right)>0$ where $d \mu$ is a fixed orientation on $\Sigma_{t}$. Besides, $A\left(e_{i}, e_{j}\right)=$ 
$h_{3 i j} e_{3}+h_{4 i j} e_{4}$ is the second fundamental form of $\Sigma_{t}$. From this and the maximum principle for parabolic equations we immediate see that being a Lagrangian local graph is preserved.

We fixed the complex structure $J^{\prime}$ that corresponds to $\omega^{\prime}$ and choose a orthonormal basis $\left\{e_{1}, e_{2}, e_{3}, e_{4}\right\}$ so that $e_{3}=J^{\prime} e_{1}$ and $e_{4}=J^{\prime} e_{2}$. The orientation given by this basis is in fact opposite to the one given by $J^{\prime}$ in the sense that $\left(\omega^{\prime}\right)^{2}\left(e_{1}, e_{2}, e_{3}, e_{4}\right)<0$. Therefore $\left(\omega^{\prime \prime}\right)^{2}\left(e_{1}, e_{2}, e_{3}, e_{4}\right)>0$ and $\eta=* \omega^{\prime \prime}$ satisfies the equation $(2.1)$.

The normal bundle of a Lagrangian surface is canonically isomorphic to its tangent bundle by $J^{\prime}$. Through this isomorphism the second fundamental form $A$ and the mean curvature vector $H$ are associated with tensors $B$ and $\sigma$. $B$ is the symmetric three-tensor defined by $B(X, Y, Z)=-\left\langle\nabla_{X} Y, J^{\prime}(Z)\right\rangle$ for $X, Y, Z \in T \Sigma$. $\sigma$ is the one-form defined by $\sigma(X)=\left\langle J^{\prime}(X), H>\right.$. If we denote $B\left(e_{i}, e_{j}, e_{k}\right)=B_{i j k}$ and $\sigma\left(e_{i}\right)=\sigma_{i}$, then $h_{3 i j}=-B_{1 i j}$ and $h_{4 i j}=$ $-B_{2 i j}$. Therefore the term involving the second fundamental form in (2.1) can be calculated as the following.

$$
\left(h_{31 k}-h_{42 k}\right)^{2}+\left(h_{32 k}+h_{41 k}\right)^{2}=\left(B_{k 11}-B_{k 22}\right)^{2}+4 B_{k 12}^{2}=2|B|^{2}-|\sigma|^{2}
$$

Therefore $\eta=* \omega^{\prime \prime}$ satisfies

$$
\frac{d}{d t} \eta=\Delta \eta+\eta\left[2|B|^{2}-|\sigma|^{2}\right]+c \eta\left(1-\eta^{2}\right)
$$

Now we proceed to prove the long time existence theorem.

Proof of Theorem A. Notice that $0 \leq \eta \leq 1$. By the equation of $\eta$ and comparison theorem for parabolic equations, we get

$$
\eta(x, t) \geq \frac{\alpha e^{c t}}{\sqrt{1+\alpha^{2} e^{2 c t}}}
$$

where $\alpha>0$ satisfies $\frac{\alpha}{\sqrt{1+\alpha^{2}}}=\min _{\Sigma_{0}} \eta$. Therefore $\eta(x, t)$ converges uniformly to 1 when $c=1$ and is nondecreasing when $c=0$. In any case, $\eta$ has a positive lower bound at any finite time.

Since

$$
|\sigma|^{2}=B_{111}^{2}+2 B_{111} B_{122}+B_{122}^{2}+B_{211}^{2}+2 B_{211} B_{222}+B_{222}^{2}
$$

and

$$
|B|^{2}=B_{111}^{2}+3 B_{112}^{2}+3 B_{122}^{2}+B_{222}^{2}
$$

It is easy to see $|\sigma|^{2} \leq \frac{4}{3}|B|^{2}$, therefore

$$
\frac{d}{d t} \eta \geq \Delta \eta+\frac{2}{3} \eta|B|^{2}+c \eta\left(1-\eta^{2}\right)
$$


We can proceed to prove regularity at any finite time as in [14]. The idea is to prove the Gaussian density

$$
\lim _{t \rightarrow t_{0}} \int \rho_{y_{0}, t_{0}} d \mu_{t}=1
$$

for any point $y_{0} \in M$ and $t_{0}<\infty$, where $\rho_{y_{0}, t_{0}}$ is the backward heat kernel

$$
\rho_{y_{0}, t_{0}}(y, t)=\frac{1}{\left(4 \pi\left(t_{0}-t\right)\right)} \exp \left(\frac{-\left|y-y_{0}\right|^{2}}{4\left(t_{0}-t\right)}\right)
$$

White's regularity theorem [16] would implies $\left(y_{0}, t_{0}\right)$ is a regular point. As in [14], for any point $\left(y_{0}, t_{0}\right)$ we can select sequences $t_{i} \rightarrow t_{0}$ and $\lambda_{i} \rightarrow \infty$ such that the parabolic rescaling of $\Sigma_{t_{i}}$ by $\lambda_{i}$ at $\left(y_{0}, t_{0}\right)$ converges to a Lagrangian submanifold with $|B|=0$, or a linear subspace. This implies the Gaussian density at $\left(y_{0}, t_{0}\right)$ is 1 and there is no singular point at $\left(y_{0}, t_{0}\right)$.

\section{Convergence at infinity-the sphere case}

In this section, we prove the convergence in the sphere case. The key point is the uniform boundedness of the norm of the second fundamental form. We accomplish this using the blow up analysis at infinity. We already have a mean curvature flow $F: \Sigma \times[0, \infty) \mapsto M$ that exists for all time. If $\sup _{\Sigma_{t}}|A|$ is not bounded, then there exists a sequence $\check{t}_{k} \rightarrow \infty$ such that $\sup _{\Sigma_{\check{t}_{k}}}|A| \rightarrow \infty$. Choose $\check{x}_{k} \in \Sigma_{\check{t}_{k}}$ such that $|A|\left(\check{x}_{k}, \check{t}_{k}\right)=\sup _{\Sigma_{\check{t}_{k}}}|A|$. Fix a number $a$ less than the injective radius of $M$. Because $M$ is compact, we may assume $\check{x}_{k} \rightarrow \check{x} \in M$ and $d_{M}\left(\check{x}_{k}, \check{x}\right)<\frac{a}{2}$ by passing to a subsequence.

Since $M$ is locally a product, we can choose a coordinate system on a neighborhood $U=U_{1} \times U_{2}$ of $\check{x}$ such that each $\omega_{i}$ on $U_{i}$ is the standard symplectic form. We shall use this coordinate system to identify $U$ with an open set $B$ in $\mathbb{R}^{4}$. On $U$ there is the metric induced from $M$ and on $B$ there is the flat metric. However, being Lagrangian does depend on any particular metric structure.

Let $S$ be the total space of the mean curvature flow $F$ in $M \times[0, \infty)$, take

$$
S_{k}=S \cap\left(U \times\left[\check{t}_{k}-1, \check{t}_{k}+1\right]\right)
$$

For any $(x, t) \in S_{k}$ denote the parabolic distance to the boundary of $U \times\left[\check{t}_{k}-\right.$ $\left.1, \check{t}_{k}+1\right]$ by

$$
\delta_{k}(x, t)=\min _{x_{0} \in \partial U, t_{0} \in\left\{\check{t}_{k}-1, \check{t}_{k}+1\right\}}\left\{d_{M}\left(x, x_{0}\right), \sqrt{\left|t-t_{0}\right|}\right\}
$$

Denote

$$
\alpha_{k}=\sup _{(x, t) \in S_{k}} \delta_{k}(x, t)|A|(x, t)
$$

Notice that $\alpha_{k}$ is a scaling invariant quantity. Since $\delta_{k}\left(\check{x}_{k}, \check{t}_{k}\right)|A|\left(\check{x}_{k}, \check{t}_{k}\right) \geq$ $\min \left\{\frac{a}{2}, 1\right\}|A|\left(\check{x}_{k}, \check{t}_{k}\right)$, we have $\alpha_{k} \rightarrow \infty$. Now we consider $S_{k}$ as a smooth flow 
in $B \subset \mathbb{R}^{4}$ with the flat metric, let

$$
\alpha_{k}^{\prime}=\sup _{(x, t) \in S_{k}} \delta_{k}^{\prime}(x, t)\left|A^{\prime}\right|(x, t)
$$

where

$$
\delta_{k}^{\prime}(x, t)=\min _{x_{0} \in \partial B, t_{0} \in\left\{\check{t}_{k}-1, \check{t}_{k}+1\right\}}\left\{\left|x-x_{0}\right|, \sqrt{\left|t-t_{0}\right|}\right\}
$$

is the parabolic distance in the flat metric on $B$ and $\left|A^{\prime}\right|(x, t)$ is the second fundamental form of $\Sigma_{t} \cap B$ as a submanifold in $\mathbb{R}^{4}$. Since the two metrics are equivalent, $\alpha_{k}^{\prime} \rightarrow \infty$ too.

Now we take $\left(x_{k}, t_{k}\right) \in S_{k}$ such that

$$
\delta_{k}^{\prime}\left(x_{k}, t_{k}\right)\left|A^{\prime}\right|\left(x_{k}, t_{k}\right) \geq \frac{\alpha_{k}^{\prime}}{2}
$$

Let $\lambda_{k}=\left|A^{\prime}\right|\left(x_{k}, t_{k}\right)$. Because $\delta_{k}^{\prime}\left(x_{k}, t_{k}\right) \leq \min \{a, 1\}, \lambda_{k} \rightarrow \infty$ too. For any $S_{k}$, we consider it as a submanifold in $B \times\left[\check{t}_{k}-1, \check{t}_{k}+1\right] \subset \mathbb{R}^{4} \times \mathbb{R}$ and take the parabolic rescaled flow $\widetilde{S}_{k}=D_{k} S_{k}$ by $\lambda_{k}$.

$$
\begin{aligned}
& D_{k}: \quad \mathbb{R}^{4} \times \mathbb{R} \quad \rightarrow \quad \mathbb{R}^{4} \times \mathbb{R} \\
& (x, t) \rightarrow\left(\lambda_{k}\left(x-x_{k}\right), \lambda_{k}^{2}\left(t-t_{k}\right)\right)
\end{aligned}
$$

Notice that $D_{k}\left(x_{k}, t_{k}\right)=(0,0)$. Let $\left|\widetilde{A}_{k}\right|(y, s)$ denote the second fundamental form of $\left(\widetilde{S}_{k}\right)_{s}$ at $y \in\left(\widetilde{S}_{k}\right)_{s}$, then $\left|\widetilde{A}_{k}\right|(0,0)=1$.

Since $\delta_{k}^{\prime}\left|A^{\prime}\right|$ is also a scaling invariant quantity.

$$
\widetilde{\delta}_{k}(0,0)\left|\widetilde{A}_{k}\right|(0,0)=\delta_{k}^{\prime}\left(x_{k}, t_{k}\right)\left|A^{\prime}\right|\left(x_{k}, t_{k}\right) \geq \frac{\alpha_{k}^{\prime}}{2}
$$

where $\widetilde{\delta}_{k}$ is the parabolic distance to the boundary of $\lambda_{k}\left(B-x_{k}\right) \times\left[\lambda_{k}^{2}\left(\check{t}_{k}-1-\right.\right.$ $\left.\left.t_{k}\right), \lambda_{k}\left(\check{t}_{k}+1-t_{k}\right)\right]$.

From this we see $\widetilde{\delta}_{k}(0,0) \rightarrow \infty$ as $k \rightarrow \infty$. For any $(y, s) \in \widetilde{S}_{k}$, $\widetilde{\delta}_{k}(y, s)\left|\widetilde{A}_{k}\right|(y, s) \leq \alpha_{k}^{\prime} \leq 2 \widetilde{\delta}_{k}(0,0)$, and therefore

$$
\left|\widetilde{A}_{k}\right|(y, s) \leq 2 \frac{\widetilde{\delta}_{k}(0,0)}{\widetilde{\delta}_{k}(y, s)} \leq 2 \frac{\widetilde{\delta}_{k}(0,0)}{\widetilde{\delta}_{k}(0,0)-\max \{|y|, \sqrt{|s|}\}}
$$

Take $(y, s) \in K$ for any compact set $K \subset \mathbb{R}^{4} \times \mathbb{R}$, the above estimate shows $\left|\widetilde{A}_{k}\right|$ is uniformly bounded for all $k$ on any compact set in space-time. Therefore $\widetilde{S}_{k} \rightarrow \widetilde{S}_{\infty}$ smoothly and since $\widetilde{\delta}_{k}(0,0) \rightarrow \infty, \widetilde{S}_{\infty}$ is defined on $(-\infty, \infty)$. We have proved the following proposition.

Proposition 3.1. If $\sup _{\Sigma_{t}}|A|$ is not bounded, then there exists a blow-up flow $\widetilde{S}_{\infty} \subset \mathbb{R}^{4} \times \mathbb{R}$ defined on the whole $(-\infty, \infty)$ with uniform bounded second fundamental form and $|A|(0,0)=1$. 
The main convergence theorem in the sphere case is the following.

Theorem 3.3.1. Under the same assumption as in Theorem A. If $M$ has positive curvature then the mean curvature flow of $\Sigma$ converges smoothly to a totally geodesic Lagrangian surface at infinity.

Proof. We already know the long time existence and we are going to show the uniform boundedness of the second fundamental form by contradiction.

By the equation of $\eta$ we have $\eta(x, t) \geq 1-\epsilon_{k}$ for $(x, t) \in S_{k}$ and $\epsilon_{k} \rightarrow 0$. This continue to hold for the corresponding $\widetilde{\eta}_{k}$ on $\widetilde{S}_{k}$. Therefore $\eta(x, t) \equiv 1$ for $(x, t) \in \widetilde{S}_{\infty} \subset \mathbb{R}^{4} \times \mathbb{R}$. In particular the $t=0$ slice is a complete Lagrangian graph with $\eta \equiv 1$. If we write the graph as $(x, y, f(x, y), g(x, y))$, then the Lagrangian condition implies

$$
f_{x} g_{y}-g_{x} f_{y}=1
$$

and $\eta=1$ is equivalent to

or

$$
\frac{2}{\sqrt{1+f_{x}^{2}+f_{y}^{2}+g_{x}^{2}+g_{y}^{2}+\left(f_{x} g_{y}-f_{y} g_{x}\right)^{2}}}=1
$$

$$
f_{x}^{2}+f_{y}^{2}+g_{x}^{2}+g_{y}^{2}=2
$$

These implies $h=f+\sqrt{-1} g$ is holomorphic and $\left|\frac{\partial h}{\partial z}\right|=1$, therefore $h$ is of the form $h=e^{\sqrt{-1} \theta} z+C$, where $\theta$ and $C$ are constants. The graph of $h$ has zero second fundamental form and this contradicts with $\left|\widetilde{A}_{\infty}\right|(0,0)=1$.

Therefore $|A|^{2}$ is uniformly bounded in space and time. By equation (7.7) in [14], $|A|^{2}$ satisfies the following equation.

$$
\frac{d}{d t}|A|^{2} \leq \Delta|A|^{2}-2|\nabla A|^{2}+4|A|^{4}+K_{1}|A|^{2}+K_{2}
$$

Integrating this equation and we see

$$
\frac{d}{d t} \int_{\Sigma_{t}}|A|^{2} d \mu_{t} \leq C
$$

Recall $\frac{d}{d t} \eta \geq \Delta \eta+\frac{2}{3} \eta|A|^{2}$ and $\eta$ has a positive lower bound, thus

$$
\int_{0}^{\infty} \int_{\Sigma_{t}}|A|^{2} d \mu_{t} d t \leq \infty
$$

Equation (3.1) and (3.2) implies

$$
\int_{\Sigma_{t}}|A|^{2} d \mu_{t} \rightarrow 0
$$

By the small $\epsilon$ regularity theorem in [4], $\sup _{\Sigma_{t}}|A|^{2} \rightarrow 0$ uniformly as $t \rightarrow \infty$. 
Since the mean curvature flow is a gradient flow and the metrics are analytic, by the theorem of Simon [11], we get convergence at infinity. The flow converges to a minimal Lagrangian submanifold with $\eta=1$. Since $\eta=*\left(\omega_{1}+\omega_{2}\right)$, this implies the limiting submanifold is holomorphic with respect to the complex structure associated with the Kähler form $\omega_{1}+\omega_{2}$. The limiting map is both holomorphic and area preserving and thus an isometry.

\section{Positive genus case}

The following theorem is a general subconvergence theorem for mean curvature flow of surfaces. The proof is essentially contained in that of Theorem 2 in [8].

Theorem 4.4.1. Let $F_{t}: \Sigma \times[0, \infty) \mapsto M$ be a smooth mean curvature flow of an immersed compact oriented surface $F_{0}(\Sigma)$ in a compact Riemannian manifold $M$. We assume $F_{0}(\Sigma)$ represents a nontrivial homology class in $M$. Then there exists a sequence $t_{i} \rightarrow \infty$ such that $F_{t_{i}}$ converges to a $C^{1, \alpha}$ branched minimal immersion $F: \widetilde{\Sigma} \mapsto M$ where $\widetilde{\Sigma}$ is a compact oriented surface and $F$ is a smooth immersion of $\widetilde{\Sigma}-B$ into $M$ for a finite set $B \subset \widetilde{\Sigma}$.

Proof. Since $\frac{d}{d t} \int d \mu_{t}=-\int|H|^{2} d \mu_{t}$, we have $\int_{0}^{\infty}\left(\int|H|^{2} d \mu_{t}\right) d t<\infty$. Therefore there exists a sequence $F_{t_{i}}$ such that $\int_{\Sigma_{t_{i}}}|H|^{2} \rightarrow 0$. Now we apply Theorem 2 of [8] with the functional $\mathfrak{F}(\Sigma)=\frac{1}{2} \int_{\Sigma}|H|^{2} d \mu-\chi(\Sigma)$ where $\chi(\Sigma)$ is the Euler number of $\Sigma$. By Gauss-Bonnet Theorem, this can be written as the form of those functionals considered in [8]. $\quad F_{t_{i}}$ form a minimizing sequence of $\mathfrak{F}$ in the space of immersions smoothly homotopic to $F_{0}$. The homology class of $F_{t_{i}}$ is nontrivial, so the diameter has positive lower bound. It follows by Simon's theorem [8] that a limit exists as a branched immersion.

The convergence is in the sense of varifold and Housdorff distance as discussed in [9]. By [10] and Definition 2 of [8], the sequence $F_{t_{i}}$, while remains a minimizing sequence for $\int|H|^{2}$, can be modified locally so that the convergence is in the following sense. There exists a sequence $\phi_{t_{i}}$ of diffeomorphisms of $\widetilde{\Sigma}-B$ onto open subsets $U_{t_{i}}$ of $\Sigma$ such that

1. $F_{t_{i}} \circ \phi_{t_{i}}$ converges to $F$ locally in the $C^{2}$ sense on $\widetilde{\Sigma}-B$.

2. $F_{t_{i}}\left(M-U_{t_{i}}\right) \subset \cup_{x \in B} B_{\epsilon_{k}}(F(x))$ for some $\epsilon_{k} \downarrow 0$.

By conclusion 2, it is not hard to see $F(\widetilde{\Sigma})$ is in the same homology class as $F_{0}(\Sigma)$. As was remark in [8], the surface $\widetilde{\Sigma}$ may have lower genus than $\Sigma$ due to necks or handles pinching. Such pinching was caused by the concentration of the limit measure $|A|^{2} d \mu_{t_{i}}$. We shall prove Theorem B now.

Proof of Theorem B. The case when $\Sigma_{1}=\Sigma_{2}=S^{2}$ is already proved in Theorem 3.3.1. We shall assume they are of positive genus now. By Theorem 4.4.1, a subsequence converges to a minimal Lagrangian immersion $F: \widetilde{\Sigma} \mapsto \Sigma_{1} \times \Sigma_{2}$ which may posses some branched points. We first show that indeed there is 
no branched point. Since a minimal immersion is a conformal harmonic map, the composite map $\pi_{1} \circ F: \widetilde{\Sigma} \mapsto \Sigma_{1}$ is a harmonic map with respect to some smooth metric in the same conformal calss as the pull back metric by $F$. This is now a degree one harmonic map since $F(\widetilde{\Sigma})$ is in the same homology class as $\Sigma_{t_{i}}$ by conclusion 2 in the remark right after Theorem 4.4.1. Since the convergence $F_{t_{i}}(\Sigma) \rightarrow F(\widetilde{\Sigma})$ is in the varifold sense and $\pi_{1} \circ F_{t_{i}}$ has positive Jacobian, $\pi_{1} \circ F$ has non-negative Jacobian. Use the proposition on page 13 of [7] we can show the Jacobian of $\pi_{1} \circ F$ is positive everywhere and $\Sigma_{\infty}$ is the graph of a map $f_{\infty}$. Therefore the sequence $\Sigma_{t_{i}}$ converges to a smooth minimal Lagrangian graph. When $\Sigma_{1}$ and $\Sigma_{2}$ are both torus, the Gauss-Bonnet theorem shows $\int|A|^{2} d \mu_{t_{i}}=\int|H|^{2} d \mu_{t_{i}} \rightarrow 0$, so the limit is totally geodesic.

This gives a new proof of the existence theorem of minimal maps between hyperbolic surfaces in Proposition 2.12 of [6].

Indeed, Theorem B also holds when $\Sigma$ is locally a graph which corresponds to the local condition $\left.\omega_{1}\right|_{\Sigma}=\left.\omega_{2}\right|_{\Sigma}>0$.

Theorem 4.4.2. Let $M=\left(\Sigma_{1}, \omega_{1}\right) \times\left(\Sigma_{2}, \omega_{2}\right)$, where $\Sigma_{1}$ and $\Sigma_{2}$ are Riemann surfaces of the same constant curvature. If $\Sigma$ is a compact Lagrangian surface with respect to $\omega_{1}-\omega_{2}$ and is locally a graph over $\Sigma_{1}$ and $\Sigma_{2}$, then the mean curvature flow of $\Sigma$ exists for all time and a sequence converges to a smooth minimal Lagrangian surface.

Proof. The locally graphical condition implies $\left.\pi_{1}\right|_{\Sigma}: \Sigma \mapsto \Sigma_{1}$ is a covering map, so $2 g-2=\operatorname{deg}\left(\left.\pi_{1}\right|_{\Sigma}\right)\left(2 g_{1}-2\right)$ where $g$ and $g_{1}$ is the genus of $\Sigma$ and $\Sigma_{1}$ respectively. Now the limit $\widetilde{\Sigma}$ has lower genus than $\Sigma$ and $\pi_{1} \circ F$ is a branched harmonic immersion of degree $\operatorname{deg}\left(\left.\pi_{1}\right|_{\Sigma}\right)$ by the the same argument. This precludes the possibility of branched point by the topological Riemann-Hurwitz formula.

We remark that the existence of minimal Lagrangian submanifold in such homology class was first proved by Y.-I. Lee using variational methods in [5].

Next we prove Theorem C. First of all, we observe that when $\Sigma_{1}=\Sigma_{2}$, the graph of the identity map is a totally geodesic submanifold in the product space. When $f: \Sigma_{1} \mapsto \Sigma_{1}$ is homotopic to the identity, we claim the limit obtained in Theorem $\mathrm{C}$ is actually totally geodesic. When $\Sigma_{1}$ is a torus, the Gauss-Bonnet theorem shows $\int|A|^{2} d \mu_{t_{i}}=\int|H|^{2} d \mu_{t_{i}} \rightarrow 0$, so the limit has $|A| \equiv 0$. When $\Sigma_{1}$ is a hyperbolic surface, we apply the uniqueness of minimal graph in each homotopy class in [6].

The next lemma should be well-known. We sketch the proof for completeness.

Lemma 4.1. Let $\Gamma$ be a totally geodesic submanifold in a Riemannian manifold of non-positive sectional curvature and $\rho(x)=d(x, \Gamma)$ is the distance function to $\Gamma$. Then $\rho$ is a convex function in a tubular neighborhood of $\Gamma$.

Proof. Let $\alpha(s)$ be a smooth curve defined for $-\epsilon<s<\epsilon$ and $\alpha(0)=x$. We need to show $\frac{d^{2}}{d s^{2}} \rho(\alpha(s)) \geq 0$. Join each point $\alpha(s)$ to $\Gamma$ by a geodesic that 
realizes the distance. The lemma now follows from the second variation formula of length (see e.g. page 20 in [1]) and the fact that $\Gamma$ is totally geodesic.

We prove Theorem $\mathrm{C}$ in the following. Corollary $\mathrm{C}$ follows from the correspondence between Lagrangian graph and area-preserving diffeomorphism.

Proof of Theorem $C$. Let $\rho$ be the distance function to the diagonal in $\Sigma \times \Sigma$. We calculate the parabolic equation of $\rho(F(x, t))$ as the following.

$$
\frac{d}{d t} \rho(F(x, t))=\nabla \rho \cdot H
$$

where $\nabla \rho$ denotes the gradient of $\rho$ in $\Sigma \times \Sigma$.

Split $\nabla \rho$ into the normal part and tangent part we get $\operatorname{div}_{\Sigma} \nabla \rho=\Delta_{\Sigma} \rho-\nabla \rho \cdot H$.

Therefore the equation is

$$
\frac{d}{d t} \rho=\Delta_{\Sigma} \rho-\operatorname{div}_{\Sigma} \nabla \rho
$$

$\operatorname{div}_{\Sigma} \nabla \rho$ is the trace of the Hessian of $\rho$ restricted to $\Sigma$ and is always nonnegative by the convexity of $\rho$. By maximum principle, $\max _{x \in \Sigma} \rho(F(x, t))$ is non-increasing. Since we already have the convergence of a subsequence $\Sigma_{t_{i}}$ in Hausdorff distance, this implies the convergence of the whole flow $\Sigma_{t}$. We can write each $\Sigma_{t}$ as $\left(x, f_{t}(x)\right)$ for $x \in \Sigma$. Since $\rho\left(\left(x, f_{t}(x)\right) \rightarrow 0\right.$ uniformly, this implies $f_{t}(x)$ converges to $x$ uniformly.

In fact, even when the domain and target of a diffeomorphism are of different conformal structure, we can prove the following isotopy theorem.

Corollary 4.1. Every area preserving diffeomorphism of Riemann surfaces is isotopic through area preserving diffeomorphisms to a minimal diffeomorphism.

Proof. Given any area preserving diffeomorphism $h: \Sigma_{1} \mapsto \Sigma_{2}$, we can compose it with another area-preserving minimal diffeomorphism $g: \Sigma_{2} \mapsto \Sigma_{1}$ so that $g \circ h$ is homotopic to the identity map on $\Sigma_{1}$. We can deform $g \circ h$ by the mean curvature flow to get $f_{t}$ isotopic to the identity map. Then $g^{-1} \circ f_{t}$ gives the desired isotopy of $h$ to $g^{-1}$.

\section{Acknowledgement}

I would like to thank Professor R. Schoen and Professor S.-T. Yau for their constant encouragement and invaluable advice. I also have benefitted greatly from the many discussion that I have with Professor G. Huisken, Professor L. Simon, and Professor B. White. 


\section{References}

[1] J. Cheeger and D. G. Ebin, Comparison theorems in Riemannian geometry, NorthHolland Mathematical Library, Vol. 9. North-Holland Publishing Co., Amsterdam-Oxford; American Elsevier Publishing Co., Inc., New York, 1975. viii+174 pp.

[2] K. Ecker and G. Huisken, Mean curvature evolution of entire graphs. Ann. of Math. (2) 130 (1989), no. 3, 453-471.

[3] G. Huisken, Asymptotic behavior for singularities of the mean curvature flow, J. Differential Geom. 31 (1990), no. 1, 285-299.

[4] T. Ilmanen, Singularities of mean curvature flow of surfaces, preprint, 1997.

[5] Y.-I. Lee, Lagrangian minimal surfaces in Khler-Einstein surfaces of negative scalar curvature, Comm. Anal. Geom. 2 (1994), no. 4, 579-592.

[6] R. Schoen, The role of harmonic mappings in rigidity and deformation problems, Complex geometry (Osaka, 1990), 179-200, Lecture Notes in Pure and Appl. Math., 143, Dekker, New York, 1993.

[7] R. Schoen and S.-T. Yau, Lectures on harmonic maps, Conference Proceedings and Lecture Notes in Geometry and Topology, II. International Press, Cambridge, MA, 1997. vi+394 pp. ISBN: 1-57146-002-0.

[8] L. Simon, Existence of Willmore surfaces Miniconference on geometry and partial differential equations (Canberra, 1985), 187-216, Proc. Centre Math. Anal. Austral. Nat. Univ., 10, Austral. Nat. Univ., Canberra, 1986.

[9] L. Simon, Existence of surfaces minimizing the Willmore functional, Comm. Anal. Geom. 1 (1993), no. 2, 281-326.

[10] L. Simon, Personal communications, April, 2001.

[11] L. Simon, Asymptotics for a class of nonlinear evolution equations, with applications to geometric problems Ann. of Math. (2) 118 (1983), no. 3, 525-571.

[12] S. Smale, Diffeomorphisms of the 2-sphere. Proc. Amer. Math. Soc. 10 1959, 621-626.

[13] K. Smoczyk, A canonical way to deform a Lagrangian submanifold., preprint, dg$\mathrm{ga} / 9605005$

[14] M-T. Wang, Mean Curvature Flow of surfaces in Einstein Four-Manifolds, to appear in J. Differential Geom.

[15] M-T. Wang, Long-time existence and convergence of graphic mean curvature flow in arbitrary codimension, to appear in Invent. Math.

[16] B. White, A local regularity theorem for classical mean curvature flow, preprint, 2000.

Department of Mathematics, Stanford University, Stanford, CA 94305.

E-mail address: mtwang@math.stanford.edu 